A practical approach to compensate for diodic effects of PS converted waves

Hengchang Dai and Xiang-Yang Li

British Geological Survey

Murchison House

West Mains Road

Edinburgh EH9 3LA

$U K$

Submitted to: The leading Edge

Address for correspondence

Dr. Hengchang Dai

British Geological Survey

Murchison House

West Mains Road

Edinburgh EH9 3LA

$U K$

Tel: +44 1316500216

Fax: +441316671877

Email: hcd@bgs.ac.uk 


\section{A practical approach to compensate for diodic effects of PS converted waves}

Hengchang Dai and Xiang-Yang Li, British Geological Survey, Edinburgh, UK

In inhomogeneous media, PS converted waves often suffer from severe diodic effects. The traveltime and amplitude of PS converted waves may be different in the forward and reverse shooting directions, giving rise to different stacking velocities of PS converted waves and velocity ratios. These effects, compounded with the asymmetric raypath of PS converted waves, will further increase the difficulties and costs in processing PS converted wave data. One common method to solve this problem is to separate a dataset into two volumes with different shooting directions (e.g. negative or positive offset directions). Different values of the PS converted wave velocities are used to process the two datasets separately and the two results are combined in the final stage. The problem with this method is that sometimes it is difficult to correlate the datasets and the final combined result may be degraded. In this paper, we propose a method to overcome this problem and apply this method to a $2 \mathrm{D}$ dataset for improving the PS converted wave imaging.

The diodic moveout of PS converted waves. In homogeneous media the moveout of PS converted waves is written as:

$$
\begin{aligned}
& t_{p s}^{2}=t_{p s 0}^{2}+\frac{h^{2}}{V_{p s}^{2}}-\frac{2 \kappa_{e f f} h^{4}}{V_{p s}^{2}\left[t_{0}^{2} V_{p s}^{2}+m_{4} \cdot h^{2}\right]}, \\
& \kappa_{\text {eff }}=\frac{\left(\gamma_{0} \gamma_{\text {eff }}-1\right)^{2}+8 \chi_{\text {eff }}\left(1+\gamma_{0}\right)}{8 \gamma_{0}\left(1+\gamma_{\text {eff }}\right)^{2}},
\end{aligned}
$$




$$
m_{4}=2 \kappa_{\text {eff }} \frac{\left(1+\gamma_{0}\right)\left[\left(\gamma_{0}-1\right) \gamma_{\text {eff }}^{2}+2 \chi_{\text {eff }}\right]}{\left(\gamma_{0}-1\right) \gamma_{\text {eff }}\left(\gamma_{0} \gamma_{\text {eff }}-1\right)+2\left(1+\gamma_{0}\right) \chi_{\text {eff }}}
$$

where $V_{p s}$ is the velocity of PS converted waves which is a combination of P-and S-wave velocities, $\gamma_{0}$ and $\gamma_{\text {eff }}$ vertical and effective $V_{p} / V_{s}$ ratios, $\chi_{\text {eff }}$ the anisotropic parameter for PS converted waves, $t_{p s 0}$ the vertical two-way travel time, $h$ the shot-receiver offset. For homogeneous media, the PS converted wave moveout is invariant when source and receiver positions are interchanged. However, in lateral inhomogeneous media, PS converted wave moveout and velocity are not invariant under an interchange of source and receiver positions. This phenomenon is called diodic velocity, a term referring to the electronic diode, which operates differently in forward and reverse directions. This phenomenon is caused by the asymmetric ray-path of PS converted waves which propagates in inhomogeneous media. Figure 1 schematically shows this phenomenon in which the media is uniform except for a zone of anomalous $\mathrm{P}$ and S velocities. For a given conversion point associated with the same offset length, the PS converted wave has different ray-paths when the source and receiver positions are changed and hence the velocities along the ray-paths are different. The two raypaths involve different P- and S-wave velocities. Hence the converted wave velocity and velocity ratio are different for the two ray-paths, and so the moveouts for the two ray-paths are different. The different moveouts increase the difficulty on processing PS converted wave data.

In PS converted wave data processing, the events should be flattened using Equation (1). However, due to the diodic effect, using one velocity model cannot flatten the events for the two datasets with opposite offset directions. 
The diodic effect also exists in prestack time migration processing. In prestack time migration, the travel time is the summation of the travel-time of the down-going P-wave and up-going S-wave.

$$
t_{p s}=t_{p}+t_{s}
$$

where $t_{p}$ and $t_{s}$ are approximated as:

$$
\begin{aligned}
& t_{p}=\frac{1}{1+\gamma_{0}} \sqrt{t_{p s 0}^{2}+\frac{w_{p}}{V_{p s}^{2}}-\frac{2 \eta_{e f f} w_{p}^{2}}{V_{p s}^{2}\left[t_{p s 0}^{2} V_{p s}^{2}+\left(1+2 \eta_{e f f}\right) w_{p}\right]}} \\
& t_{s}=\frac{\gamma_{0}}{1+\gamma_{0}} \sqrt{t_{p s 0}^{2}+\frac{w_{s}}{V_{p s}^{2}}-\frac{2 \xi_{e f f} w_{s}^{2}}{V_{p s}^{2}\left[t_{p s 0}^{2} V_{p s}^{2}+w_{s}\right]}}
\end{aligned}
$$

Where $\quad w_{p}=\frac{x_{p}^{2}\left(1+\gamma_{0}\right)\left(1+\gamma_{\text {eff }}\right)}{\gamma_{\text {eff }}}, \quad w_{s}=\frac{x_{s}^{2}\left(1+\gamma_{0}\right)\left(1+\gamma_{\text {eff }}\right)}{\gamma_{0}}, \quad \eta_{e f f}=\frac{\chi}{\left(\gamma_{0}-1\right) \gamma_{e f f}^{2}} \quad$ and $\xi_{\text {eff }}=-\frac{\chi}{\gamma_{0}-1} \cdot x_{p}$ is the distance from the shot to image point, $x_{s}$ the distance from image point to receiver. Similar to stacking processing, for a common image point, when the positions of source and receiver are changed, the ray paths are different and involve different velocities. Due to the diodic effect, the events in the common image point gather cannot be flattened using one velocity model for the two datasets with opposite offset directions.

To overcome this problem, two velocity models should be used. One model is used for the positive offset data and the other is used for the negative offset data. Conventionally, the dataset is separated into two volumes according to the offset direction. Each volume is processed using its own velocity model. However, there are some drawbacks which prevent this method being applied to real datasets. One of the drawbacks is that it is difficult to align the events when the two datasets are separately processed, especially when the signal-to- 
noise ratio is low or many events need to be aligned. Therefore, when the two poorly aligned datasets are combined, the final results are degraded. It is necessary to overcome this drawback. One solution is to process the two datasets in one interface, but using two velocity models for the opposite offset directions.

The perturbation of the velocity of PS converted waves. The velocity model of PS converted waves has four parameters $\left(V_{p s}, \gamma_{0}, \gamma_{\text {eff }}\right.$ and $\left.\chi_{\text {eff }}\right) . V_{p s}, \gamma_{\text {eff }}$ and $\chi_{\text {eff }}$ might have different values in the two models. Thus seven parameters will need to be estimated. This is not practical because the uncertainty and workload are very large. Fortunately, the parameters in PS converted wave velocity model have different sensitivities to moveout. The error in the moveout is mainly determined by the PS converted wave velocity, $V_{p s}$. Other parameters have less effect on the moveout. This means we may need two velocities, but other parameters can be unchanged for the opposite offset data. We also find that in practice, we can express the two velocities as a base velocity and a perturbation:

$$
\begin{aligned}
& V_{p s+o}=V_{p s}(1+\Delta) \\
& V_{p s-o}=V_{p s}(1-\Delta)
\end{aligned}
$$

where $V_{p s}$ is the base velocity defined as the velocity without diodic effects, and $\Delta$ is the velocity perturbation (as a percentage).

Figure 2 schematically shows how to apply two velocities to correct a diodic moveout. If only the base velocity is used to correct the event, the corrected event becomes a dipping straight line and the resulting stacked event will smear. When we add the velocity perturbation, the corrected event becomes a horizontal line and the resulting stacked event 
will be better resolved. The advantage of this perturbation method is that it can decouple the diodic moveout into two parts. One part is related to the base velocity and one part is related to the perturbation. The base velocity is used to correct the curved part of the events and the perturbation is used to correct the dipping part of the events. Following the above analysis, we developed tools to perform the diodic velocity analysis and apply the diodic velocity to correct the diodic moveout. We will discuss the tools in the following section.

In prestack time migration, we use a similar perturbation method to deal with the diodic travel-times. The two velocities for opposite offset directions are also expressed as Equations (7) and (8). Note that in prestack time migration, an asymmetric travel time may be caused by incorrect values of the effective velocity ratio. However, if we assume that the values of the effective velocity ratio in prestack time migration are equal to the values in the moveout, and also that the effect of the effective velocity ratio and anisotropy on travel time is smaller than the velocity, we do not need to add a perturbation to the effective velocity ratio and anisotropy. We also developed a GUI tool for migration velocity analysis, and a prestack time migration tool to compensate the diodic moveout in prestack time migration.

Data example. We applied the tools to a dataset which is from a 2D/4C marine acquisition from a field located approximately 60km offshore North Africa. This area is partially obscured by a gas chimney that causes heavy distortion. There is an outer seabed collapse zone with an inner elevated, hard ground feature which leads to the channel being obscured. The gas in the media attenuates the $\mathrm{P}$-wave energy and reduces the $\mathrm{P}$-wave velocity. However, it has a small effect on shear-wave velocity. The inhomogeneity caused by the gas results in the diodic moveout. Without a suitable method to compensate for the diodic effect, 
the image obtained from this dataset will be degraded. Here we apply our tools to the dataset to deal with the diodic movout.

This perturbation approach is firstly applied to the NMO+stacking procedure. Figures 3 and 4 show snapshots of the velocity analysis tool which performs the diodic velocity analysis. In both figures, the first left panel shows the PS converted wave velocity, the second shows the velocity ratio, the third shows the anisotropy and the fourth shows the velocity perturbation. The furthest right is the gather panel. Without the perturbation, the events become dipping after the NMO correction using the base velocity only (Fig 3). With the perturbation considered, the events became horizontal (Fig 4). Stacking the horizontal events yield a more focused imaging than stacking the dipping events. Figure 5 and 6 show a comparison between the images with and without velocity perturbation. Figure 5 shows the sallow part of the images between $2 \mathrm{~s}$ and $4 \mathrm{~s}$. Two images have similar structures but the events obtained with velocity perturbation applied are clearer and have stronger energies. Figure 6 shows the deep part of the image between 5.5s and 7.5s. Although the main features a similar, the structures are different. The events obtained with velocity perturbation applied have stronger energies.

We also apply this perturbation approach to prestack Kirchhoff time migration. Figure 7 and 8 show snapshots of the migration velocity tool to perform the diodic effect analysis. In Figure 7, without the velocity perturbation, the events are dipping, similar to those in Figure 3. In Figure 8, with the velocity perturbation, the events become horizontal. Stacking the horizontal events will yield a more focused imaging than stacking the dipping events.

Figure 9 and 10 show the parts of final migrated images with and without velocity perturbation. Figure 9 shows the sallow part of the migrated images between $2 \mathrm{~s}$ and $4 \mathrm{~s}$. Two 
images have similar structure; however the images with velocity perturbation are clearer and have larger contrast. Figure 10 shows the deep part of the migrated image between 5.5 and 7.5s. The improvement in the image with velocity perturbation is obvious. For example, the image obtained with velocity perturbation has larger contrast than that without velocity perturbation. Note that the appearances of the structure of the two images are different. Events in Figure 10a are dipping and those in Figure 10b are horizontal.

Discussions. From the above processed results, we find that the velocity perturbation method can improve the quality of the image in both $\mathrm{NMO+stacking} \mathrm{and} \mathrm{prestack} \mathrm{time} \mathrm{migration}$ procedures. However, we must note that this improvement is obtained from compensating diodic moveout only. In the NMO+stacking procedure, because the velocity perturbation is applied after asymptotic conversion-point binning, it does not affect the location of the conversion point of PS converted waves. However, in prestack time migration, the velocity perturbation method does have an effect on the location of scatter point of PS converted waves. If we compare the stacking sections (Figures 5 and 9) and migration images (Figures 6 and 10), we can see significant differences between the two results. The reason is that in stacking processing, the asymptotic conversion point binning procedure cannot accurately locate the conversion points, but the prestack migration can accurately locate the image point. The quality of the migrated image improved more than the quality of the stacked sections. The velocity perturbation has larger effect in prestack time migration. For example, the events are more continues and clearer in Figure 10 (b) than in Figure 10 (a). 
Conclusions. We have proposed a practical approach to compensate the diodic moveout of PS converted waves using a velocity perturbation method. GUI tools have been developed to perform the diodic velocity analysis for stacking procedure and prestack time migration, and command line tools perform the diodic moveout correction and prestack time migration with diodic moveout compensation. In this method, the diodic moveout is decoupled into two parts. One part is related to the base velocity and the other part is related to the velocity perturbation. The base velocity is used to correct the curved feature of the diodic moveout and the velocity perturbation is used to correct the dipping feature of the diodic moveout. Application of these tools to a real dataset has shown that we can easily estimate the base velocity and the velocity perturbation. The events in negative and positive offset data can be easily aligned, and the quality of both the final stacked image and migrated image are improved.

Suggested reading. “A simplified moveout formula for P-, S- and converted-waves in multilayered media” by Dai and Li. (67 $7^{\text {th }}$ EAGE meeting, Madrid, Spain, Extended Abstracts. 2005). “Compensating for the Effects of Gas Clouds on C-wave Imaging: A Case Study from Valhall”. By Li, Dai, Mueller, and Barkved (The Leading Edge 20. 2001). “Converted wave and convertion-point equations in layered VTI media: theory and application by Li. and Yuan. (Journal of Applied Geophysics, 54, 2003). “Converted wave reflection seismology over inhomogeneous, anisotropic media” by Thomsen. (Geophysics 64, 1999). 
Acknowledgements. This work is supported by the Edinburgh Anisotropy Project (EAP) of the British Geological Survey, and is published with the permission of the Executive Director of the British Geological Survey (NERC) and the EAP sponsors. 


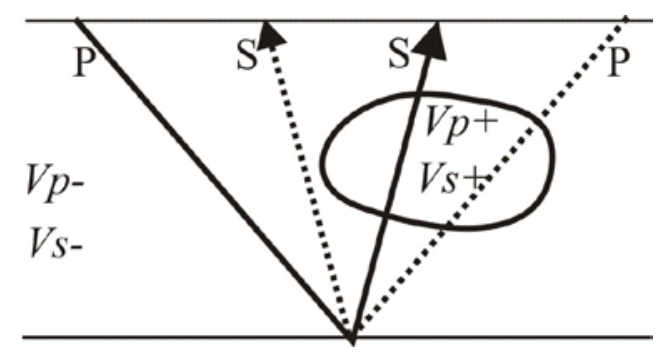

Figure 1. PS converted wave ray-paths in an inhomogeneous medium.

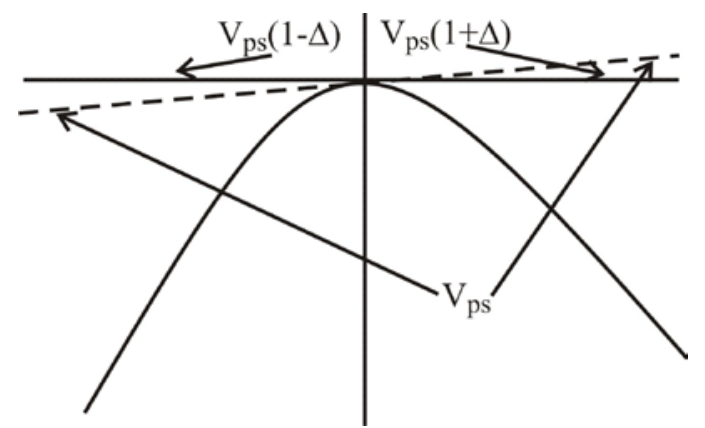

Figure 2. Diodic moveout correction by using different velocities for two opposite offset directions. 


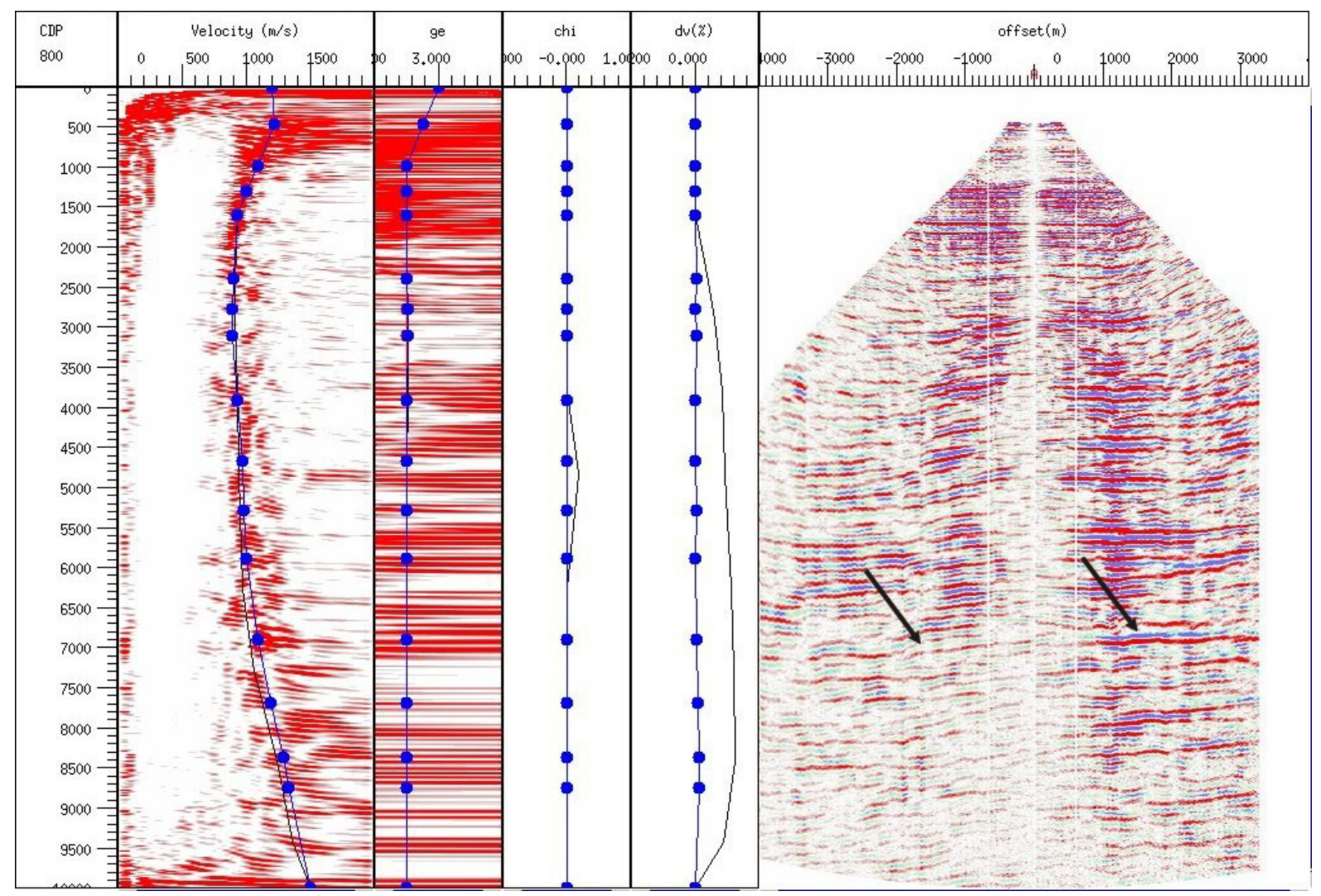

Figure 3. Snapshot of diodic velocity analysis tool. With velocity perturbation $\Delta=0.0$, the event becomes dipping after NMO correction using the same velocity model for two opposite offset direction datasets. 


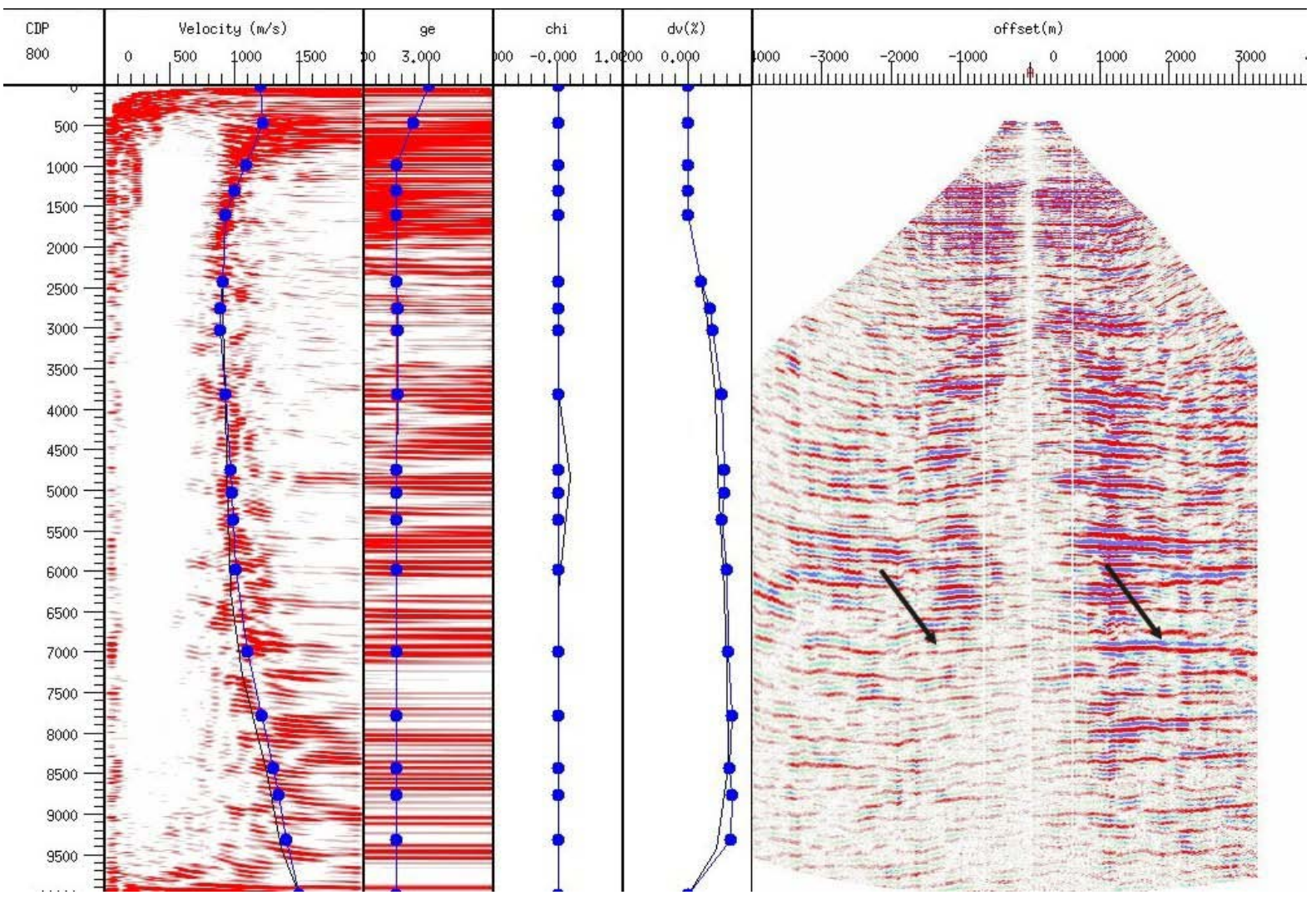

Figure 4. Snapshot of diodic velocity analysis tool. With a perturbation applied, the events between $5 \mathrm{~s}$ and $6 \mathrm{~s}$, become horizontal after NMO correction.
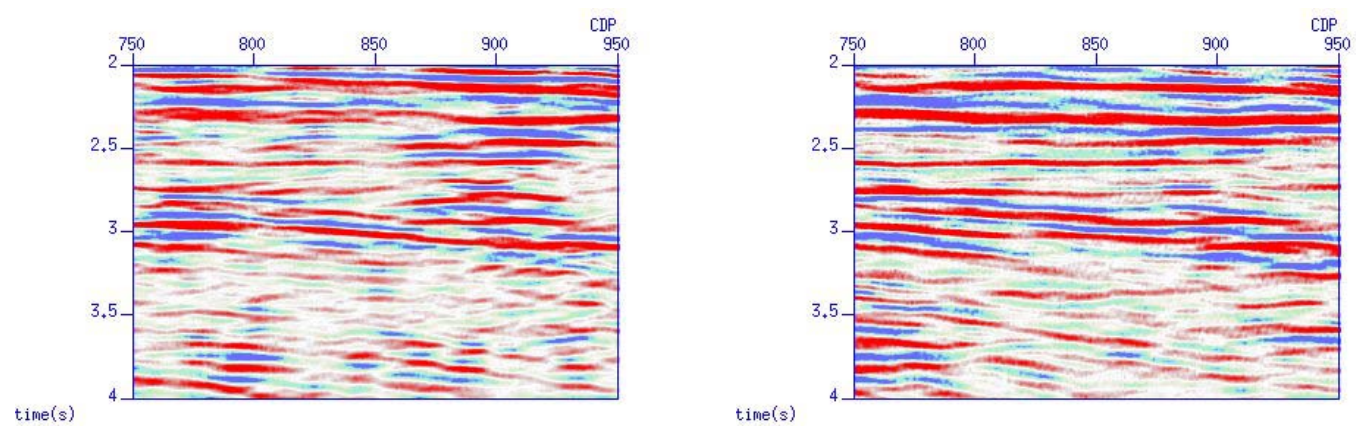

Figure 5: Sallow part of Stack sections (a) without and (b) with velocity perturbation. 


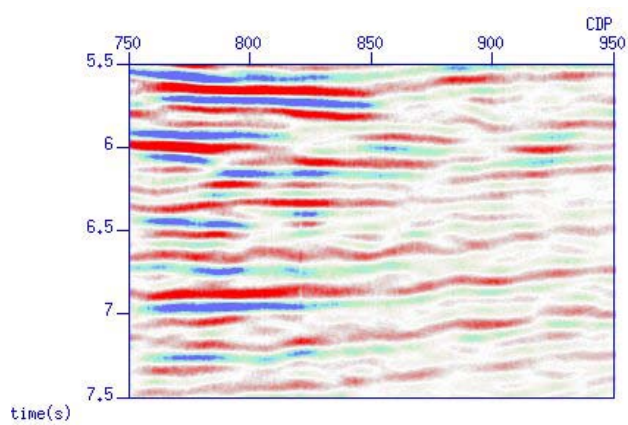

(a)

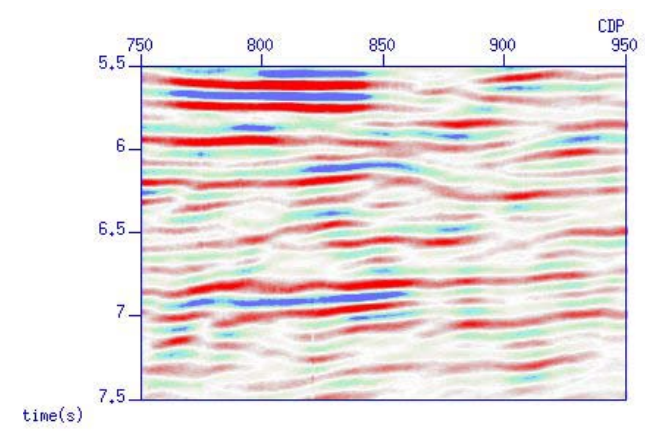

(b)

Figure 6: Deep part of Stack sections (a) without and (b) with velocity perturbation.

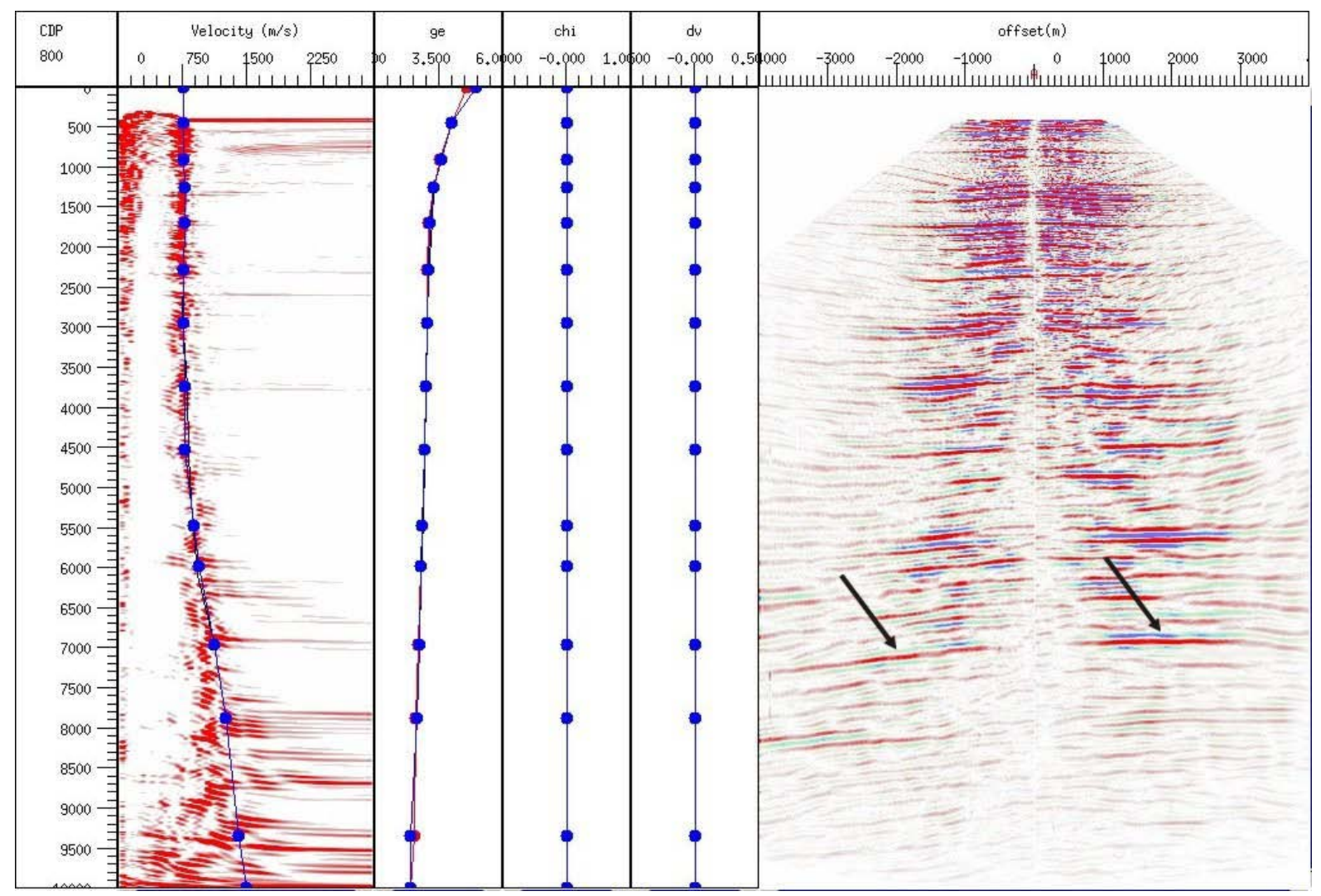

Figure 7. Snapshot of migration diodic velocity analysis tool. With velocity perturbation $\Delta=0.0$, the event becomes dipping after NMO correction using the same velocity model for two opposite offset direction datasets. 


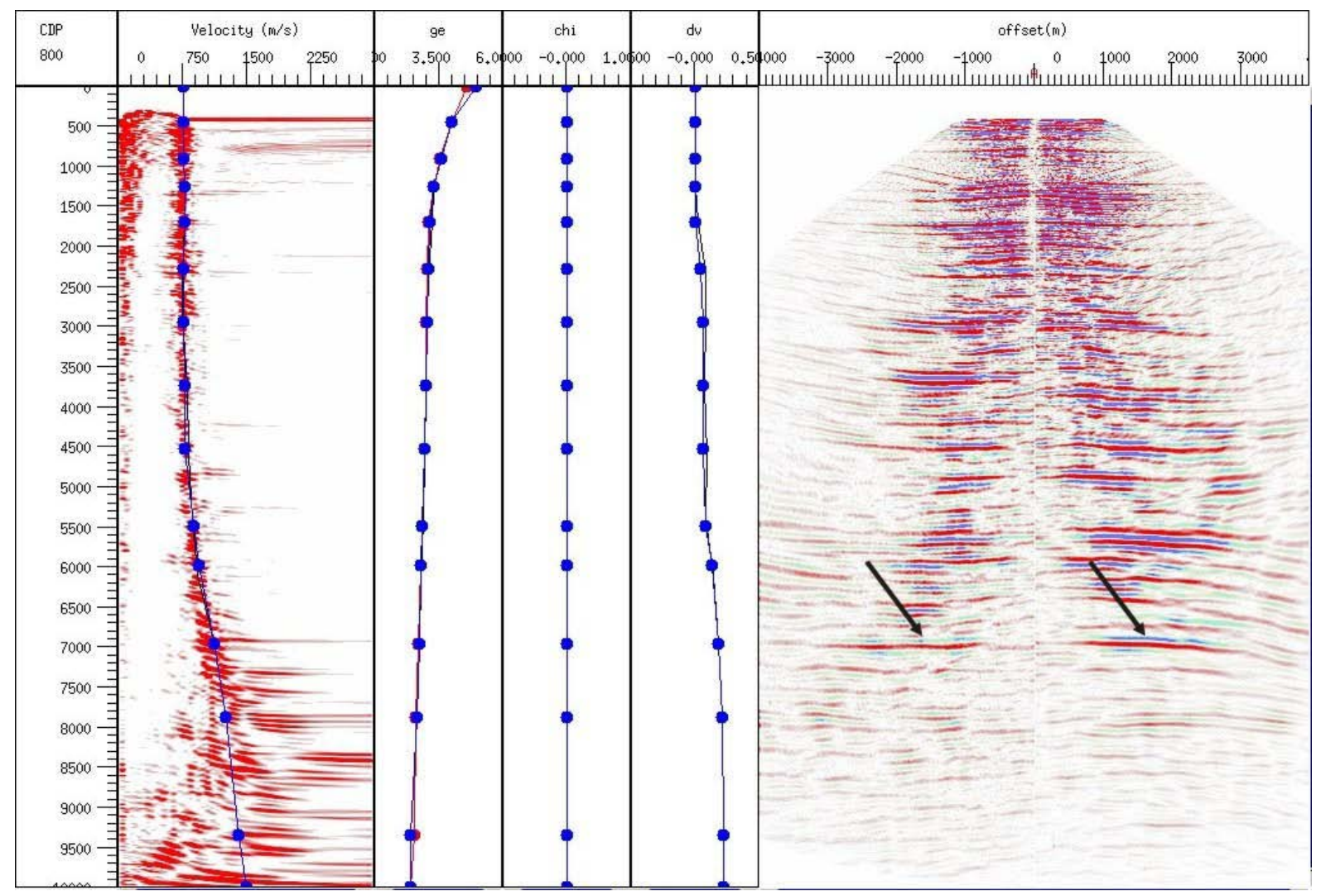

Figure 8. Snapshot of diodic velocity analysis tool. With a perturbation applied for the events between 5 s and 6s, the events become horizontal after NMO correction 


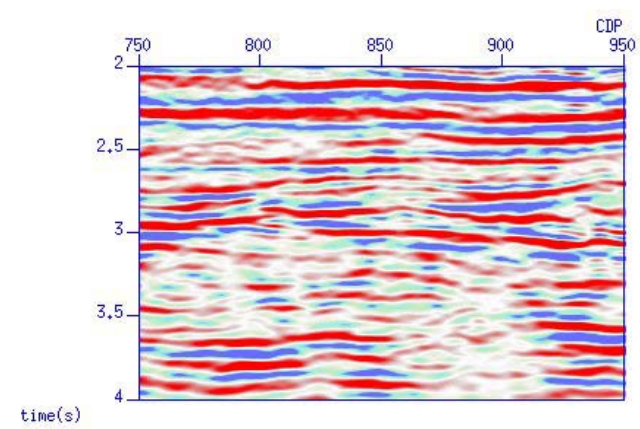

(a)

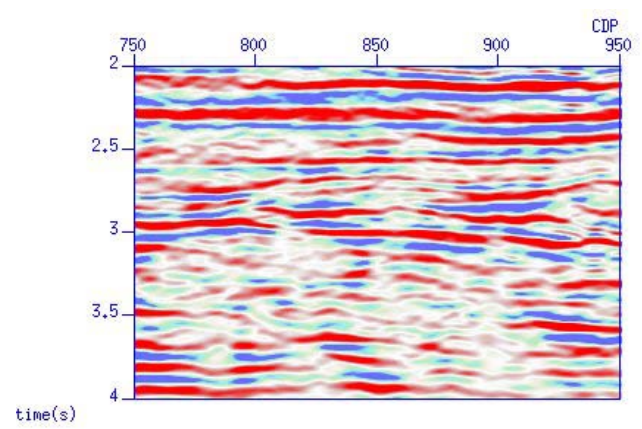

(b)

Figure 9. Sallow part of migrated images (a) without and (b) with velocity perturbation.

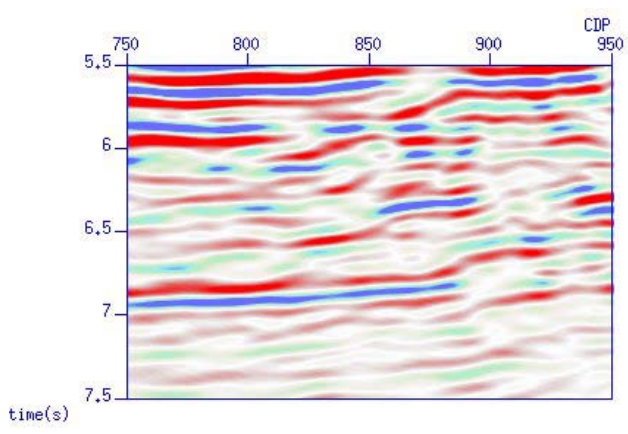

(a)

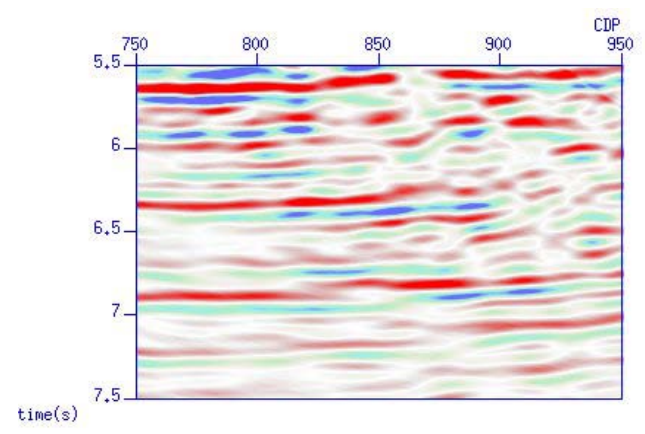

(b)

Figure 10. Deep part of migrated images (a) without and (b) with velocity perturbation. 